\title{
Tägliche Mundhygiene mit Spezial-Zahnpasten und Mundspülllösungen
}

Der Spezialist für Mund- und Zahnpflegeprodukte CP GABA lud, gemeinsam mit dem Prophylaxe Team Club und dem FVDZ Bayern, erneut zum Prophylaxe-Symposium ein. Rund 300 Teilnehmer kamen nach Würzburg. Sie informierten sich zum Thema „Praktische Parodontologie - und mehr!" und erhielten neue Anregungen für ihre tägliche Arbeit am Patienten. Auch dieses Jahr hat sich CP GABA für eine dialogbasierte Veranstaltung entschieden, die von den Teilnehmern rege angenommen wurde. Referiert wurde über Mundspüllösungen am Arbeitsplatz als eine effektive Prävention und über Zahnpasten als Hilfsmittel mit wichtiger (zahn) medizinischer Wirksamkeit. Ferner wurde berichtet, dass extrinsische Verfärbungen reversibel und durch effektive häusliche Mundhygiene zu vermeiden sind und dass sich mit verschiedenen Fluoridverbindungen ein sehr viel größeres Spektrum abdecken lässt als nur die Prävention von Karies. Die abschließende interaktive
Expertenrunde mit den Referenten (PD Dr. Gregor Petersilka,

Prof. Dr. Stefan Zimmer, PD Dr. Cornelia Frese, Dr. Karolin Höfer) zeigte das hohe Interesse der Profession an allen Themen des Symposiums. Übereinstimmend kamen die Teilnehmer gemeinsam mit den Experten zum Schluss, dass eine individuelle, fallbezogene Empfehlung zur häuslichen Mundhygiene die beste Lösung für die Zahn- und Mundgesundheit ihrer Patienten ist. Information und Anmeldung zu weiteren Fortbildungsveranstaltungen unter www.cpgaba-events.de

Nach einer Pressemitteilung der CP GABA GmbH, Hamburg www.gaba-dent.de 